\title{
CONTRIBUCIÓN DE EXPERIENCIAS DE VINCULACIÓN SOCIAL DE LAS UNIVERSIDADES AL MEJORAMIENTO DE LA CALIDAD ACADÉMICA Y FACTORES QUE LIMITAN SU DESARROLLO Y VALORACIÓN INSTITUCIONAL
}

\author{
Daniel Mato *
}

\begin{abstract}
*Doctor en Ciencias Sociales, Universidad Central de Venezuela, Investigador Principal del Consejo Nacional de Investigaciones Científicas y Técnicas (CONICET) adscripto al Centro Interdisciplinario de Estudios Avanzados de la Universidad Nacional Tres de Febrero. Argentina. E-mail: dmato2007@gmail.com
\end{abstract}

Resumen: Este artículo examina las contribuciones al mejoramiento de la calidad académica logradas mediante experiencias extracurriculares de colaboración entre equipos universitarios y comunidades y organizaciones sociales, así como algunos factores que limitan su mayor desarrollo y valoración por parte de las universidades. La investigación toma como referencia más de doscientas experiencias desarrolladas por equipos de unas cuarenta universidades públicas argentinas, constituidos por docentes y estudiantes de diversas disciplinas, en diversos contextos sociales, las cuales según los casos son conceptualizadas por sus protagonistas en términos de extensión, vinculación social, aprendizaje-servicio, investigación-acción, o voluntariado. El elemento común a todas estas experiencias es que fueron realizadas con apoyo del Programa de Voluntariado Universitario de la Secretaría de Políticas Universitarias, del Ministerio de Educación de la República Argentina. La investigación permite concluir que estos tipos de experiencias no solo contribuyen a mejorar la calidad de vida de sectores sociales, sino, también la formación profesional ofrecida por las universidades, así como la calidad de investigación que realizan dichos equipos. Sin embargo, estos tipos de actividades no suelen contar con valoración institucional. Esto hace aconsejable que las universidades y los organismos de evaluación integren estas modalidades de trabajo como un componente importante de los planes de estudio, y le otorguen mayor valoración en sus políticas de investigación y de carrera docente. Si bien, esta investigación toma como referencia algunas experiencias de universidades argentinas, el problema no se limita a ese país, pues se enmarca en algunos procesos de alcance internacional, que se expresan en ciertas culturas institucionales, en algunas políticas y en la creciente ascendencia de los rankings universitarios internacionales.

Palabras clave: Educación superior. Evaluación. Calidad. Investigación. Extensión. Vinculación social. Colaboración intercultural. Comunicación intercultural. Argentina.

\section{CONTRIBUIÇÃO DE EXPERIÊNCIAS DE VINCULAÇÃO SOCIAL DAS \\ UNIVERSIDADES PARA O MELHORAMENTO DA QUALIDADE ACADÊMICA E FATORES QUE LIMITAM SEU DESENVOLVIMENTO E VALORIZAÇÃO INSTITUCIONAL}

Resumo: Este artigo examina as contribuições para o melhoramento da qualidade acadêmica alcançadas mediante experiências extracurriculares de colaboração entre equipes universitárias e comunidades e organizações sociais, assim como alguns fatores que limitam seu maior desenvolvimeto e valorização por parte das universidades. A pesquisa toma como referência mais de duzentas experiências desenvolvidas por equipes de umas quarenta universidades públicas argentinas constituídas por docentes e estudantes de diversas disciplinas, em diversos contextos sociais, as quais conforme os casos são conceptualizadas por seus protagonistas em termos de extensão, vinculação social, aprendizagem-serviço, pesquisa-ação, ou voluntariado. O elemento comum a todas estas experiências é que foram realizadas com apoio do Programa de Voluntariado Universitário da Secretaria de Políticas Universitárias, do Ministerio de Educação da República Argentina. A pesquisa permite concluir que estes tipos de experiências não só contribuem para melhorar a qualidade de vida de 
setores sociais, como também para a formação profissional oferecida pelas universidades, assim como a qualidade de pesquisa que referidas equipes realizam. Contudo, estes tipos de atividades não costumam contar com valorização institucional. Isto torna aconselhável que as universidades e os organismos de avaliação integrem estas modalidades de trabalho como um componente importante dos programas de estudos e lhe atribuam maior valorização em suas políticas de pesquisa e de carreira docente. Ainda que esta pesquisa tome como referência algumas experiências de universidades argentinas, o problema não se limita a esse país, pois se enquadra em alguns processos de alcance internacional, que se expressam em certas culturas institucionais, em algumas políticas e na crescente ascendência dos rankings universitários internacionais.

Palavras-chave: Educação superior. Avaliação. Qualidade. Extensão. Vinculação social. Colaboração intercultural. Comunicação intercultural. Argentina.

\title{
CONTRIBUTION OF UNIVERSITY SOCIAL OUTREACH EXPERIENCES TO THE IMPROVEMENT OF ACADEMIC QUALITY AND FACTORS WHICH LIMIT THEIR DEVELOPMENT AND INSTITUTIONAL VALUATION.
}

\begin{abstract}
This article discusses some contributions to the improvement of academic quality achieved by university teams through their extra-curricular activities carried out in collaboration with communities and social organizations. It also examines some factors that limit its further development and institutional appreciation. The research is based on over two hundred experiences, from forty Argentine public universities, developed by teams constituted by professors and students from different disciplines, some of them interdisciplinary, in varied social contexts. Some of these experiences are conceptualized by their participants in terms of university extension, social reach, service learning, action-research, or university voluntary work. Their common characteristic is that all of them have received support from the University Voluntary Program of the Secretary of University Policies, of the Ministry of Education of Argentina. The research leads to conclude that this kind of experiences not only contribute to enhancing the quality of life of involved social groups, but also the professional training provided by universities, as well as the quality of the research carried by these teams. Nevertheless, experiences of this kind usually are not institutionally valued. This makes advisable that universities and university evaluation agencies include this orientations of work as an important element of university activities, as well as that they enhance the value attribute to them in research policies and teacher performance assessment. Despite this research is based on some experiences developed from Argentine universities, the problem in question is not limited to this country, because it is related to some worldwide social processes, which are associated to certain institutional cultures, some policies, and the increasing impact of International University League Tables.
\end{abstract}

Key Words: Higher education. Evaluation. Quality. Research. University outreach.

Este artículo se basa en una investigación de mayor alcance ${ }^{1}$ sobre ciertos tipos de aprendizajes que equipos de docentes y estudiantes de universidades públicas argentinas han logrado mediante experiencias extra-curriculares realizadas con comunidades y organizaciones sociales, así como acerca de algunos factores que afectan su desarrollo y/o su reconocimiento y valoración. Lo aquí

1 Este artículo expone algunos resultados del proyecto de investigación "Comunicación y aprendizajes interculturales de equipos universitarios en experiencias con comunidades y organizaciones sociales en el marco del Programa de Voluntariado Universitario (PVU) de la Secretaría de Políticas Universitarias (2008-2010)" que el autor realiza en tanto Investigador Principal del Consejo Nacional de Investigaciones Científicas y Técnicas (CONICET) y de la Universidad Nacional Tres de Febrero (UNTREF), de Argentina, contando para ello con el apoyo institucional y económico de ambas instituciones. 
expuesto se basa principalmente en el análisis demás de doscientas experiencias desarrolladas por equipos de unas cuarenta universidades públicas argentinas, constituidos por docentes y estudiantes de diversas disciplinas, en disímiles contextos sociales, las cuales según los casos son conceptualizadas por sus protagonistas en términos de extensión, vinculación social, aprendizaje-servicio, investigación-acción, o voluntariado. El elemento común a todas estas experiencias es que recibieron apoyo del Programa de Voluntariado Universitario (PVU) de la Secretaría de Políticas Universitarias, del Ministerio de Educación de la República Argentina, en las convocatorias realizadas en los años 2008 o 2010, según los casos².

No obstante, debe señalarse que ni este artículo, ni la investigación de la que resulta, son sobre el PVU. Su foco de análisis lo constituyen los aprendizajes resultantes de las experiencias de colaboración realizadas por equipos universitarios con diversos sectores de población y las tensiones institucionales y problemas de comunicación intercultural (con actores extra-universitarios, pero especialmente al interior de las instituciones: entre culturas universitarias diferentes entre sí) en cuyo marco se desarrollan. La relación del presente texto con el PVU se limita a que las experiencias objeto de este estudio han recibido apoyo económico de dicho programa. Si bien esta investigación puede brindar insumos interesantes para apreciar el PVU, ella no basta para evaluar una política pública de tan amplio alcance ${ }^{3}$.

2 La realización de esta investigación ha sido posible gracias al apoyo de la Secretaría de Políticas Universitárias del Ministerio de Educación de la República Argentina y especialmente al equipo del Programa de Voluntariado Universitario, que facilito la información básica para la misma y los contactos com las y los docentes responsables de los proyectos estudiados.

3 El PVU fue puesto en marcha por la Secretaría de Políticas Universitarias en marzo de 2006. Desde entonces cada año realiza una convocatoria destinada a seleccionar proyectos de equipos constituidos por docentes, investigadores y estudiantes universitarios a los cuales brinda un modesto apoyo económico para realizar sus propuestas que deben estar orientadas, combinadamente, tanto a mejorar la calidad de vida de la población, y especialmente de las comunidades con mayores necesidades insatisfechas, como a favorecer la formación profesional de los estudiantes y el trabajo académico. Desde su creación en 2006 y hasta 2010 el PVU realizó cinco convocatorias, mediante las cuales otorgó apoyo a 2.370 proyectos de equipos universitarios orientados combinadamente a mejorar la calidad de vida de la población, y especialmente de las comunidades con mayores necesidades insatisfechas, y a favorecer la formación profesional de los estudiantes y el trabajo académico. Estos proyectos han sido desarrollados por equipos de 48 universidades, los cuales han involucrado la participación de casi 50.000 estudiantes, más de 9.600 docentes y más de 4.600 organizaciones sociales. Adicionalmente, desde 2009 el PVU ha realizado una segunda convocatoria anual para proyectos de voluntariado universitario orientados específicamente a fortalecer las capacidades de escuelas secundarias. La investigación que da lugar a este artículo no incluye el estudio de experiencias incluidas en esta segunda convocatoria (cuyo número no ha sido incluido en las referencias cuantitativas antes mencionadas). Estudiar una política pública de tan amplio alcance escapa a las posibilidades de esta investigación, cuya única relación con el PVU se limita al hecho práctico que su existencia ha hecho posible tener acceso a cierta información general sobre estos tipos de experiencias y ha facilitado el contacto directo con un número significativo de equipos universitarios que han recibido su apoyo. Sin este apoyo, la investigación que da lugar a este artículo no hubiera sido posible, pues en el país se desarrolla un número indeterminado de experiencias extracurriculares con comunidades y 
En cambio, la investigación en que se basa el presente artículo responde al interés de revisar críticamente la plausibilidad de ciertas "ideas academicistas"(nótese el uso del sufijo "istas", para destacar que se trata de una cierta orientación de valores y modelo universitario de referencia) que en líneas generales se expresan en términos de que la calidad de las universidades descansa en buena medida en la formación de profesionales que responda a las demandas del mercado de empleo (y no necesariamente a demandas de diversos sectores sociales, ni a posibilidades de emprendimientos individuales o colectivos que respondan a las mismas), y en la realización de investigaciones que den lugar a publicaciones en revistas académicas "arbitradas" por pares (o "dictaminadas", según el vocabulario en uso en algunos países), de gran circulación internacional y alto "impacto". En el marco de las mencionadas ideas, el "impacto" se mide por la cantidad de citas y/o referencias a los artículos publicados en revistas académicas que se registran en artículos de otros autores publicados también en revistas académicas.

La inquietud por someter a análisis la plausibilidad de las mencionadas "ideas academicistas" se asienta principalmente en los resultados obtenidos mediante la realización de dos investigaciones de cierto alcance. La primera de ellas, cuyos principales resultados han sido analizados anteriormente en varias publicaciones por lo cual en estas páginas se hará sólo muy somera referencia a los mismos, ha estado enfocada en el estudio de aproximadamente cuarenta experiencias de educación superior orientadas principalmente a responder a demandas y propuestas de comunidades y organizaciones indígenas y afrodescendientes de once países de América Latina. Algunas de estas experiencias corresponden a programas especiales desarrollados en universidades "convencionales" y otras a universidades $u$ otras instituciones de educación superior enteramente concebidas como "interculturales" (MATO, 2011; MATO, 2008a, 2008b, 2009b, 2009c). La segunda investigaciones la ya mencionada en el primer párrafo de este artículo, la cual toma como referencia más de doscientas experiencias desarrolladas por equipos de unas cuarenta universidades públicas argentinas, constituidos por docentes y estudiantes de diversas disciplinas, en disímiles contextos sociales, las cuales según los casos son conceptualizadas por sus protagonistas en términos de extensión, vinculación social, aprendizaje-servicio, investigación-acción, o voluntariado.

organizaciones sociales, a cargo de numerosos equipos en todas las universidades públicas nacionales y no existe un banco de datos al respecto. Además del apoyo que brinda el PVU, algunas experiencias se desarrollan con apoyo de las respectivas instituciones y/o de otros programas estatales de carácter nacional o provincial. 
Los principales resultados de esta segunda investigación se presentan y analizan en las próximas secciones de este artículo. Mientras tanto, sintéticamente cabe adelantar que éstos conducen a concluir que estos tipos de experiencias, no solo contribuyen a mejorar la calidad de vida de los sectores sociales involucrados, sino también la formación profesional ofrecida por las universidades, así como las posibilidades y calidad de investigación que realizan dichos equipos. Sin embargo, estos tipos de actividades no suelen contar con valoración institucional, pues pese a algunas transformaciones recientes, las actividades que en el lenguaje universitario institucional argentino suelen encuadrarse como de "extensión", continúan recibiendo menor valoración institucional que las de investigación. A esto se añade la escasa valoración por las mismas de parte de sectores universitarios que adscriben a otro modelo de universidad, así como la incidencia de algunos factores externos, como la creciente importancia de los rankings universitarios internacionales en la formación de opinión pública general y en sectores universitarios y de formulación de políticas y presupuestos.

\section{TENSIONES Y CONFLICTOS RESPECTO DE LA IDEA DE "CALIDAD ACADÉMICA"}

La existencia de problemas asociados a políticas y programas de estímulo a la investigación basados en indicadores cuantitativos que no valoran las otras funciones universitarias y la creciente incidencia de los rankings universitarios internacionales en la formación de opinión de actores significativos no son exclusivos de las universidades argentinas(DIAS SOBRINHO; BRITO DIAS, 2006, LÓPEZ SEGRERA, 2008, ORDORIKA, 2007).

El hecho que en las universidades convivan y contiendan diversos "modelos ideales" de universidad no es nuevo, ni tiene porqué resultar sorprendente. Algunos sectores universitarios están más orientados a la conocida imagen de la "torre de marfil", según los casos, más o menos aislada del contexto social, mientras que otros lo están a la idea de que estas instituciones forman parte de contextos sociales específicos a los cuales deben servir de manera directa y de los cuales pueden y deben aprender a aprender. Pero, estas diferencias y tensiones internas se han visto agudizadas especialmente por la incidencia de dos tipos de factores extrauniversitarios, los cuales, además, han ido encontrando formas institucionales de existencia y operación dentro de las propias universidades.

Uno de estos factores está asociado a políticas de agencias gubernamentales de los respectivos países, independientemente de que en el origen u orientación de las mismas pueda identificarse alguna incidencia de organismos interguber- 
namentales y/o multilaterales y de cooperación internacional. Mientras que, el otro es de carácter privado y transnacional.

El primer factor es que en varios países latinoamericanos se han creado programas de "estímulo a la investigación" que, entre otras cosas, han procurado acentuar la dedicación a la investigación por parte de los docentes universitarios, otorgando diversos tipos de apoyos económicos a las actividades de investigación y/o directos a los investigadores. En algunos países estos programas de estimulo a la investigación han sido creados por las agencias nacionales de ciencia y técnica. En otros, los ministerios, secretarías u otros organismos rectores de la educación superior han creado agencias de evaluación y/o acreditación de las universidades que entre otros indicadores dan especial importancia al número e impacto de publicaciones de los docentes de las mismas, y relativamente menos a sus labores de extensión y/o vinculación social.

Ambos tipos de políticas y programas suelen evaluar logros mediante índices que, entre otras variables, miden la producción de graduados de las instituciones y la productividad de investigación de los docentes. En este último caso lo hacen en términos de artículos publicados en revistas académicas "arbitradas" (o "dictaminadas", según el vocabulario en algunos países) por pares, de gran circulación internacional y alto "impacto". Así, se otorgan beneficios económicos y de ascenso o calificación a los docentes en función de la cantidad de sus publicaciones en revistas académicas y el "impacto" de las mismas medido en términos citas en resistas del mismo tipo. El problema es que esos índices son tomados como si fueran la calidad misma. Lo que parece expresar la calidad educativa en los procesos de evaluación y acreditación usuales se reduce a la demostración cuantitativa de desempeños y resultados de graduación de profesionales, cantidad de publicaciones y citas de las mismas, rendimiento estudiantil y otros productos cuantificables (DIAS SOBRINHO; BRITO DIAS, 2006, p. 12).

A ese primer conjunto de factores nacionales, más recientemente se ha agregado un factor de carácter transnacional. Se trata del hecho que diversas empresas privadas que trabajan a escala internacional han desarrollado rankings internacionales de universidades. Estos son construidos con base en indicadores ad hoc, que otorgan especial valoración tanto a las publicaciones de sus docentes en revistas académicas de circulación internacional y alto impacto (lo cual otorga preeminencia a las publicaciones en inglés), como al éxito de sus egresados en conseguir empleo. El caso es que, gracias al papel de las empresas de medios de comunicación masiva, los rankings internacionales de universidades vienen teniendo creciente impacto en la formación de opinión pública en general, así 
como en la de sectores universitarios, y de tomadores de decisiones políticas y presupuestarias respecto de la educación superior.

El papel de los medios al respecto no es nada despreciable, e incluso adquiere tonos bastante sensacionalistas con alta capacidad de impacto en la opinión pública. Por ejemplo, el 9 de septiembre de 2010, BBC mundo generó una noticia cuyo título fue "Ninguna universidad de A. Latina entre las mejores 200 del mundo", en la cual exponía el ranking presentado en el estudio anual elaborado por la empresa consultora privada británica QS. En la nota se explica que QS construye su ranking a partir de una clasificación en la cual pondera "en un $40 \%$ la reputación académica, $10 \%$ la empleabilidad, en un $20 \%$ a las publicaciones académicas de los profesores, el $20 \%$ a la tasa de estudiantes por profesor y dan un 10\% adicional según el carácter internacional de la composición de profesores y alumnos" (Disponible en:

$<$ http://www.bbc.co.uk/mundo/cultura_sociedad/2010/09/100909_ranking_universidades_qs_unam_rg.shtml $>$. Acceso en: 15 oct. 2012).

Noticias de este tipo son repetidas por medios impresos y electrónicos de la región, e incluso sirven de base para notas de opinión de columnistas que son citados en múltiples medios. Por ejemplo, el 6 de octubre de 2012, el Nuevo Herald, publicó una nota firmada por el periodista Andrés Oppenheimer, quien desde 1983 escribe para The Miami Herald y su columna se edita semanalmente en más de cincuenta periódicos de Estados Unidos y América latina. Esta nota, que se basa en otro ranking, el Times HigherEducationWorldUniversity Ranking, titulada "Malas noticias para las universidades latinoamericanas", (Disponible en: http://www.elnuevoherald. com/2012/10/06/1316638/malas-noticias-para-universidades.html, Acceso en: 9 oct. 2012), fue reproducida en varios periódicos latinoamericanos, entre otros, por ejemplo, en La Nación, de Buenos Aires. En esa nota el mencionado periodista sostiene:

[...] pocos repararon en una noticia que debería haber producido alarma: un nuevo ranking de las mejores universidades del mundo revela una ausencia casi total de instituciones latinoamericanas. El Times Higher Education World University Ranking, que consigna las 400 mejores universidades del mundo y que fue dado a conocer en Londres el 3 de octubre, revela que, pese al hecho de que Brasil es la sexta economía del mundo y México la decimocuarta, no hay una sola universidad latinoamericana entre las 100 mejores y hay apenas cuatro entre las 400 mejores (Disponible en: <http://www. lanacion.com.ar/1515519-malas-noticias-para-las-universidadeslatinoamericanas>. Acceso en: 9 oct. 2012) 
Los rankings internacionales y su proyección a través de las empresas de medios de comunicación no sólo tiene incidencia en la opinión pública en general, sino que también parecen estar teniendo importancia al interior de las universidades. Esto explica porque, el 6 de mayo de 2011, el IV Encuentro de Redes Universitarias y Consejos de Rectores de América Latina y el Caribe en la Ciudad de Buenos Aires, se hizo eco del tema, y aprobó por unanimidad un documento titulado "Posición de América latina y el Caribe ante los rankings de la Educación Superior". Este documento, basado no sólo en los intercambios sostenidos en dicha reunión, sino también en una extensa y bibliografía internacional que se refiere al final del mismo, entre otras cosas sostiene que existen "efectos indeseables de la difusión de rankings pues se presiona la orientación de las decisiones de política universitaria o de cambio institucional, a partir de la opinión pública que genera; y no en función de un claro proyecto institucional" (Disponible en: www.iesalc.unesco.org.ve/.../posicion_alc_ante_rankings. pdf Acceso en: 9 oct. 2012; en página 6 del documento en pdf)

Ese mismo documento puntualiza que la mayoría de estos rankings "han sido desarrollados ya sea por empresas editoriales (diarios o revistas), o por grupos de consultoría independientes". Adicionalmente señala que

Mediante los rankings, centrados en los resultados de la investigación y la medición de citas y publicaciones, se instala una nueva escala de prestigio, la cual resulta en gran medida dominada por las universidades líderes anglo-americanas y se convierte en el medio de asignar posiciones en los circuitos productores de bienes de conocimiento y de identificar sus flujos de transmisión. En este proceso se distingue la producción científica, reconocida y codificada, de otras formas de conocimiento. También se establece una división entre universidades productoras de conocimiento científico, consideradas jugadoras legítimas en la economía del conocimiento, e instituciones fundamentalmente para la formación profesional. (Disponible en: www. iesalc.unesco.org.ve/.../posicion_alc_ante_rankings.pdf. Acceso en: 9 oct. 2012; en página 5 del documento en pdf).

El caso es que las orientaciones academicistas pre-existentes en las universidades descriptas anteriormente, se ven crecientemente reforzadas por la incidencia de estos dos tipos de factores, en cuyo marco la idea de calidad académica está fuertemente asociada a indicadores cuantitativos de publicaciones en revistas académicas y su impacto en otras publicaciones semejantes, antes que a la generación de conocimientos útiles y/o a la formación de profesionales y desarrollo de soluciones en función de las necesidades y demandas provenien- 
tes de los contextos sociales de los cuales las universidades forman parte. La excepción a este criterio suele limitarse a la generación de tecnologías que sean "patentables" (ya que el indicador utilizado en este caso suele ser "patentes" generadas), lo cual es prácticamente inaplicable a las humanidades y ciencias sociales y/o a la "transferencia de conocimientos" al -así llamado- "sector productivo", que son otros dos indicadores de "calidad" de las universidades que suelen aceptarse desde las orientaciones academicistas. La transferencia de conocimientos a otros sectores sociales; la coproducción de conocimientos con, y/o la generación de respuestas prácticas a las demandas y propuestas de, diversos tipos de comunidades; y/o la identificación, valoración, aplicación, y/o proyección, de conocimientos propios de diversas comunidades o actores sociales hacia otros ámbitos sociales (incluido el académico); no suelen ser logros que desde las mencionadas orientaciones "academicistas" se tomen en cuenta para evaluar la "calidad" de las universidades. Se trata en definitiva de una interpretación particular de qué se entiende por "calidad académica" que está fuertemente asociada a una particular visión del papel de las universidades, la cual se ha hecho hegemónica y se sostiene un tanto tautologicamente, con la fuerza que suele tener el "sentido común", o los sistemas de creencias, lo cual se expresa en un conjunto de indicadores cuantitativos de evaluación.

En contraste con ese sistema de creencias, las preguntas que han orientado la investigación sobre más de doscientas experiencias desarrolladas por equipos de unas cuarenta universidades públicas argentinas, en la cual se basa principalmente el presente artículo, derivan de los resultados obtenidos en la otra investigación antes mencionada, cuyo alcance geográfico es más amplio. Viene al caso comentar sucintamente los resultados de dicha investigación precedente, debido a que contribuyen a hacer plausible que las observaciones y resultados expuestos en el presente artículo podrían resultar significativos más allá de los marcos institucionales de sus referentes empíricos inmediatos: el mencionado conjunto de experiencias desarrolladas en Argentina.

Ese otro proyecto de más amplio alcance geográfico al que se hace referencia es el Proyecto Diversidad Cultural e Interculturalidad en Educación Superior en América Latina, el cual fue creado en 2007 por el Instituto Internacional de la UNESCO para la Educación Superior en América Latina y el Caribe (UNESCO-IESALC). Este proyecto está dedicado a documentar y analizar experiencias de instituciones de educación superior (IES) de América Latina orientadas a responder a necesidades, demandas, y propuestas de investigación y de formación en educación superior formuladas por comunidades indígenas y afrodescendientes. Con la colaboración de aproximadamente setenta colegas de 
once países latinoamericanos, hasta la fecha el Proyecto ha logrado documentar la existencia de más de un centenar de experiencias en el campo que le concierne $\mathrm{y}$ ha realizado estudios particulares sobre aproximadamente cuarenta de ellas, además de estudios sobre los respectivos contextos nacionales (MATO, 2011; MATO, 2008a, 2009b, 2009c, 2012).

Lo que aquí interesa destacar de ese proyecto precedente es que esos estudios han permitido concluir que las universidades interculturales creadas, según los casos, por organizaciones indígenas o afrodescendientes, o por agencias gubernamentales, así como los programas de orientación intercultural creados al interior de universidades "convencionales" (entendidas simplemente como aquellas que no están explícitamente orientadas a responder a necesidades, demandas y propuestas de pueblos indígenas y afrodescendientes) y otros programas creados mediante alianzas entre universidades "convencionales" y organizaciones indígenas y/o afrodescendientes, se caracterizan por estar fuertemente vinculados a sus respectivos contextos locales. Esto se expresa, entre otras cosas, en que ajustan su oferta educativa a necesidades, demandas y proyectos provenientes de los contextos sociales en que actúan y la relacionan no solo con oportunidades de empleo (especialmente locales y regionales subnacionales), sino también con la generación de iniciativas productivas y de servicio a la comunidad. Otra característica saliente de estas experiencias de educación superior es que desarrollan modalidades participativas de aprendizaje, frecuentemente centradas en la investigación aplicada, así como que integran aprendizaje, investigación y servicio a las comunidades. Así mismo, se caracterizar por integrar diversos tipos de saberes y modos de producción de conocimiento, por el interés en generar desarrollos tecnológicos basados en conocimientos propios de las comunidades con las cuales trabajan, así como por formar profesionales orientados a contribuir al desarrollo sostenible local y regional y al mejoramiento de la calidad de vida de sus comunidades (MATO, 2011; MATO, 2008a, 2009b, 2009c, 2012).

Las mencionadas conclusiones de ese proyecto precedente llevaron a pensar que las universidades "convencionales" podían aprender mucho de esas modalidades de diseño curricular tan vinculadas a las necesidades, demandas y propuestas de sus respectivos contextos sociales. Adicionalmente, estimularon a este autor a investigar sobre las experiencias de "colaboración intercultural" de equipos de universidades "convencionales" con comunidades y organizaciones sociales, incluso aunque éstas no fueran indígenas o afrodescendientes, que es lo que ha dado lugar a la ya mencionada investigación más reciente en la cual se basa principalmente este artículo. 
Llegados a este punto corresponde especificar que en esta investigación la expresión "colaboración intercultural" se utiliza, en sentido amplio y abierto, para caracterizar las formas de colaboración entre actores sociales que respecto de los asuntos objeto de la colaboración en cuestión se perciben mutuamente, o resulta plausible caracterizar, como culturalmente diferentes entre sí. Aunque sólo sea de manera muy sintética parece necesario apuntar que este uso del concepto de "cultura" no limita su aplicación a casos de diferencias étnicas, nacionales, religiosas o lingüísticas, sino que lo extiende para abarcar también casos usualmente conceptualizados en términos de culturas profesionales, institucionales, territoriales, de clase, de género, de generación, o cualesquiera otras que resulten significativas para los asuntos respecto de los cuales establecen tales relaciones de colaboración. Este uso del concepto de "cultura" se basa en la aproximación socio-semiótica a la idea cultura, en cuyo marco este término se entiende, no como una "cosa" (sustantivo), sino como una herramienta de análisis que permite dar cuenta de modalidades de producción, circulación, apropiación y transformaciones de "sentido" por parte de actores sociales (CUCHE, 1999, GARCÍA CANCLINI, 2004, MATO, 2007, entre otros). Por otra parte, la aplicación propuesta de la idea de "colaboración intercultural" se apoya en la revisión crítica de la idea de "interculturalidad" y sus aplicaciones sobre la que hemos expuesto en publicaciones anteriores (MATO, 2009a, 2011, 2012) y ensayado en una investigación de campo precedente (MATO; MALDONADO; REY 2011).

Con base en lo antes expuesto, en la investigación en que se basa el presente artículo se utiliza la idea de "colaboración intercultural" para analizar las experiencias de trabajo de equipos universitarios con comunidades y organizaciones sociales. Este uso responde a que los casos de referencia hacen plausible afirmar que en tales relaciones unos encaran el trabajo desde los conocimientos y marcos interpretativos propios de sus culturas disciplinares (además de hacerlo desde las que puedan tener como individuos), incluso aunque frecuentemente lo hagan desde posicionamientos críticos dentro de ellas, y los otros lo hacen desde sus particulares culturales étnicas, territoriales, de clase, de género, de generación, o la que sea según los casos de referencia, pero, en todo caso no universitarias, ni académicamente disciplinares. Por otra parte, la idea de "comunicación intercultural" se utiliza para analizar ciertos aspectos de las experiencias estudiadas, porque ésta resulta fértil para comprender situaciones de comunicación tanto entre actores universitarios y no-universitarios, como entre diversos sectores universitarios que manejan disímiles representaciones del "papel de la universidad", las cuales según los 
casos se expresan en mayor, menor, o ningún interés por el trabajo con actores sociales extra-universitarios.

Dada la polarización y controversias existentes entre los partidarios de uno y otro modelo de universidad, no parece fructífero limitar la revisión crítica acerca de las ideas "academicistas" de "calidad" a una contraposición de argumentos de principio respecto de las supuestas bondades o perjuicios de que las universidades e instituciones de investigación se vinculen proactivamente con comunidades y organizaciones sociales, no solo para responder a sus necesidades y demandas, sino también para aprender de ellas. Por ello, la investigación en que se basa este artículo está orientada a recabar y analizar información acerca de cómo las experiencias de trabajo de equipos universitarios con diversos sectores de población, adicionalmente a su capacidad de favorecer mejoras en la calidad de vida de dichos sectores como usualmente se procura, contribuyen a la obtención de nuevos conocimientos y destrezas por parte de estudiantes y profesores, así como a identificar nuevas necesidades y/o intereses de investigación y/o de formación, y en algunos casos incluso también a desarrollar investigaciones que -según los casos- se expresan en tesis de los estudiantes y publicaciones de los docentes.

\section{ALCANCE DE LA INVESTIGACIÓN REALIZADA}

Los avances de investigación que se comentan a continuación se basan en el análisis de datos obtenidos mediante dos tipos de fuentes. Por un lado, en el examen de los Informes Finales de 150 proyectos de 22 universidades nacionales, de los 353 que fueron aprobados en la Convocatoria del PVU del año 2008. Por otro, en el estudio de las respuestas a cuestionarios especialmente diseñados para esta investigación que fueron emitidas por los responsables de un cierto número de proyectos aprobados en dos convocatorias del PVU. Más concretamente, se trata de las respuestas formuladas por los responsables de 58 de los 353 proyectos apoyados por el PVU mediante la Convocatoria del año 2008, en este caso correspondientes a 20 universidades, así como por las respuestas a los mismos cuestionarios dadas por los responsables de 150 proyectos de los 510 aprobados en la Convocatoria del año 2010, en este caso correspondientes a 39 universidades ${ }^{4}$.

4 Cabe aclarar que no se trata de muestras escogidas al azar, sino que en ambos casos los cuestionarios de esta investigación fueron enviados a la totalidad de responsables de proyectos aprobados en cada Convocatoria cuyas direcciones electrónicas fueron suministradas por el PVU, las cuales en el caso del 2008 fueron solo 137 de las 353 de esa Convocatoria, mientras que en la del 2010 fueron la totalidad del respectivo universo. Tomando estas cifras en consideración cabe afirmar que en el caso de 2008 los cuestionarios 
Antes de avanzar en la exposición de los resultados de la investigación, conviene apuntar que el PVU no sólo ha hecho posible el desarrollo de nuevas experiencias extracurriculares generalmente conceptualizadas explícitamente en términos de "voluntariado universitario", sino que además ha apoyado económicamente y ha contribuido a valorizar la labor de numerosos equipos universitarios que ya venían trabajando -en algunos casos desde hace varios años-con comunidades y organizaciones sociales, generalmente conceptualizando su trabajo en términos de extensión, vinculación social, servicio a la comunidad, y aprendizaje-servicio. Algunas de estas experiencias anteriormente han recibido apoyo de las respectivas universidades, otras lo han recibido de otros programas nacionales o provinciales, y no pocas de ellas se han realizado sin apoyo económico de ningún tipo, sino gracias al compromiso y empeño de sus protagonistas.

\section{PRINCIPALES CONCLUSIONES DE LA INVESTIGACIÓN REALIZADA}

El análisis de los mencionados informes finales de los proyectos y de las respuestas a los cuestionarios enviados a los responsables de los mismos, permite observar que las experiencias desarrolladas, por los equipos universitarios con comunidades y organizaciones sociales, no solamente han contribuido de diversas maneras al mejoramiento de la calidad de vida de éstas, sino que, además, mediante su realización, los equipos universitarios han logrado asegurar un conjunto de logros académicamente significativos:

a) Mejorar la formación de los estudiantes, como, por ejemplo, mediante el desarrollo de diversos tipos de destrezas prácticas, puesta en práctica de conocimientos adquiridos en aula, adquisición de nuevos conocimientos, desarrollo de perspectivas críticas respecto de conocimientos teóricos, desarrollo de destrezas de investigación, entre otros.

b) Identificar aspectos de la "realidad" no previstos en los programas de formación, y que sería conveniente incluir en los programas de las asignaturas y/o planes de estudio para lograr resultados más efectivos.

c) Comprender la complejidad de problemas que no pueden resolverse desde perspectivas unidisciplinares.

de esta investigación fueron respondidos por el $42 \%$ de los responsables de proyectos consultados, y en el caso de 2010 fueron respondidos por $29 \%$ de los responsables a quienes se les envío el respectivo Cuestionario. Estos porcentajes resultarían mayores si en cada caso se considera que un cierto número de direcciones electrónicas estaban erradas, o el respectivo buzón estaba completo y la consulta no llegó a ser vista por el respectivo responsable del proyecto. 
d) Desarrollar capacidades para el trabajo interdisciplinario

e) Adquirir nuevos conocimientos y destrezas útiles para el desarrollo de labores docentes y/o para el ejercicio profesional.

f) Avanzar en el desarrollo de proyectos de investigación existentes

g) Identificar nuevas necesidades o intereses de investigación y plantear nuevos proyectos de investigación.

h) Identificar la existencia y valoración de saberes no académicos que permiten enriquecer la formación profesional.

i) Identificar la existencia entre la población de algunos prejuicios y creencias que afectan la práctica profesional propia de las disciplinas de los miembros de los equipos universitarios participantes.

Por otra parte, el análisis de los referidos informes finales y de las respuestas a los mencionados cuestionarios ha permitido identificar tres tipos principales de dificultades de "comunicación intercultural" en el desarrollo de estos proyectos:

a) Las asociadas a diferencias entre las culturas disciplinarias y las de los sectores de población con los cuales se desarrollaron las experiencias.

b) Las asociadas a diferencias entre las diversas culturas disciplinarias.

c) Las asociadas a diferencias entre las culturas institucionales propias de las universidades y las de otras instituciones públicas (por ej.: municipios, gobernaciones, escuelas, hospitales).

d) Las asociadas a diferencias entre los valores y visiones del papel de la universidad de los equipos que desarrollan estas experiencias y los prevalecientes entre otros sectores de las mismas universidades (según los casos otros equipos docentes, o bien funcionarios y autoridades universitarias).

Para no quedarnos en el nivel de las generalizaciones parece conveniente ilustrar algunos de esos logros y dificultades refiriendo algunos ejemplos concretos de de las experiencias de trabajo de equipos universitarios con comunidades y organizaciones sociales.

\section{EJEMPLOS DE ALGUNOS APRENDIZAJES CONCRETOS REALIZADOS EN LAS EXPERIENCIAS CON COMUNIDADES Y ORGANIZACIONES SOCIALES}

En esta sección se transcriben fragmentos de las respuestas de los responsables de algunos de los proyectos analizados a preguntas acerca de si estas 
experiencias habían facilitado la identificación de necesidades e formación o investigación, y/o el desarrollo de aprendizajes en los equipos universitarios participantes. Los nombres e instituciones de pertenencia de los responsables de los proyectos se citan con su debida autorización.

La profesora Marcela Irene Coppola, de la Facultad de Ciencias Veterinarias de la Universidad de Buenos Aires, a cargo de un proyecto de Producción Ovina y Desarrollo Rural que recibió apoyo del PVU en 2008,respondió que la experiencia de vinculación les permitió conocer más en profundidad la problemática del grupo con el cual trabajaron, y que además:

[...] permitió incorporar en la planificación la transmisión de resultados de investigaciones realizadas en la Facultad con anterioridad al proyecto, pero también motivó la realización de nuevas investigaciones (acerca del estatus parasitológico y mapa de características de la lana, teñido de la lana con tintes naturales, aspectos identitarios para el desarrollo de productos con aplicación de diseño)'”. [Sostuvo, además, que la experiencia permitió articular] "contenidos curriculares con su aplicación a un caso real. Para los docentes fue enfrentarlos a una situación de producción familiar, no a escala a la cual estaban acostumbrados.

La profesora María Cristina Ojeda de Gómez, titular de la Cátedra de "Odontología Social y Comunitaria", de la Carrera de Odontología de la Universidad Nacional de Entre Ríos, a cargo de un proyecto de voluntariado con poblaciones de las etnias toba y mocoví, rurales y urbanas, que recibió apoyo del PVU en 2008,apuntó:

Se registraron no solo necesidades sentidas de las familias (trabajo, educación, alimentos), sino inequidad y discriminación sobre la cultura aborigen, no de parte de las instituciones, sino de los criollos y gringos que comparten los espacios geográficos. [Y adicionalmente señaló:] Se registraron y sistematizaron datos, que luego fueron presentados en jornadas científicas institucionales. Los mismos también fueron utilizados para abrir líneas de investigación, se concretaron 2 temas de tesis para acceder a un doctorado en Odontología, y 4 becas de iniciación en la investigación de jóvenes egresadas.

La profesora Nilda Dora Vignale, de la Facultad de Ciencias Agrarias de la Universidad Nacional de Jujuy, que fue responsable del proyecto "Conociendo, documentando, y socializando saberes tradicionales sobre las plantas alimenticias y medicinales nativas de la Quebrada de Humahuaca", que recibió apoyo del PVU en 2008, así como del proyecto "Recuperando y revalorizando el patrimonio cultural andino desde la percepción del hombre por las plantas que conforman su entorno natural”, que lo recibió en 2010, señaló: 
[La experiencia] permitió intensificar la formación en etnobotánica, campo multidisciplinario que estudia las relaciones del hombre con las plantas que integran su entorno, tanto en carreras del área de la Biología como de Agronomía. Por ejemplo, el estudio de las plantas alimenticias y medicinales de la quebrada de Humahuaca desde este abordaje posibilita tener en cuenta aspectos de la medicina tradicional. [...] El desarrollo de talleres con los jóvenes estudiantes de la Escuela de Alternancia de El Moreno sobre las especies nativas medicinales ha permitido conocer, no solamente nuevos usos de algunas de las plantas que integran la farmacopea andina, sino ampliar saberes acerca de las percepciones sobre la flora.

La Dra. Iris Aybar, de la Cátedra de Patología de la Facultad de Medicina de la Universidad Nacional de Tucumán, a cargo del proyecto "Nuevos caminos para derribar barreras y prevenir el cancer de mama a través de expresiones artísticas", que recibió apoyo del PVU en 2010, en sus respuestas al Cuestionario sostuvo:

A partir de los proyectos de extensión desarrollados por la cátedra se realizaron investigaciones destinadas a poner de manifiesto las representaciones sociales del cáncer genitomamario con la finalidad de mejorar la cobertura en prestaciones preventivas. Los intereses en formación se volcaron al área de la comunicación para favorecer el éxito de las intervenciones, ya que una de las principales dificultades fueron las diferencias de intereses entre los que haciamos el proyecto y quienes eran sus beneficiarios. Ej: Se incorporaron talleres de comunicación a alumnos y docentes en el último voluntariado [...] Tanto el equipo de docentes profesionales como los estudiantes participantes recibieron capacitaciones dentro del proyecto y las pusieron en práctica en el terreno. La adquisición de un lenguaje cientifico técnico adecuado y la transformación en un lenguaje familiar a la comunidad que no distorsione el mensaje, es quizá uno de los hechos más relevantes. Por otro lado, el médico tiende a deshumanizar la práctica por lo que "la escucha" de inquietudes y la visualización y creación de expresiones artísticas vinculadas a la temática desnuda la representación de la comunidad frente al cáncer. El comprender el concepto de representación social y vivenciarlo en las diferentes actividades realizadas por la comunidad de mujeres, orienta al médico a efectuar ajustes en sus decisiones de planificación en salud (prevención). La fluidez de comunicación permite la gestión más efectiva del caso.

El profesor César Enrique Sirka, de la Facultad de Recursos Naturales de la Universidad Nacional de Formosa, responsable de un proyecto de producción agropecuaria y forestal, que recibió apoyo del PVU en 2010, señaló que el desarrollo del proyecto

[...] ha permitido adquirir nuevos conocimientos y destrezas, plasmados en trabajo de investigación y propuestas de tesis elaborados por alumnos con orientación 
de los docentes con temas que surgidos de las necesidades y realidades socio productivas locales. [...] las experiencias desarrolladas a través del programa de voluntariado universitario han posibilitado interacciones con el medio socio productivo local, [..., y] potenciar el desarrollo rural de ciertas actividades donde se pueden conjugar el conocimiento académico con el conocimiento tradicional a efectos de mayor comprensión de problemas existentes.

La Dra. Betina Cecilia Pezzani, de la Cátedra de Microbiología y Parasitología de la Universidad Nacional de La Plata, a cargo de un proyecto de control de las parasitosis intestinales y nutrición, que recibió apoyo del PVU en 2010, se expresó en los siguientes términos:

Nuestro grupo de trabajo viene desarrollando diversos proyectos que integran activamente a los estudiantes universitarios que manifiestan un gran entusiasmo. La participación de estudiantes voluntarios ha aumentado año a año, vemos un gran interés en sumarse a proyectos que demanda un compromiso con la comunidad. [...] La mayoría de ellos han manifestado que su participación en trabajos con la comunidad les permitió tanto el reconocimiento "en terreno" de las condiciones culturales y sanitarias de los pobladores de bajos recursos económicos como corroborar "en la práctica" lo que leen en los libros de texto que utilizan para estudiar Medicina. La experiencia comunitaria les permitió tomar conciencia del compromiso social que tienen como futuros profesionales de la salud. En varias ocasiones han manifestado la necesidad de incorporar este tipo de actividades en el plan de la carrera de medicina, entienden que es necesario que la Universidad se acerque a la comunidad a través de este tipo de actividades estableciendo puentes de comunicación que benefician tanto a docentes y estudiantes como a la propia comunidad a través de un trabajo organizado y enmarcado formalmente. Ellos mismos comentan que no son "los mismos" antes y después de haber participado en el proyecto. [...] Para los estudiantes universitarios significa un entrenamiento donde lo humano es prioritario. Entender al otro como un par, humanizar el trato con el otro, escucharlo y entenderlo. Para los docentes además, fue también una práctica interactuar con las autoridades de la Unidad Sanitaria y las escuelas. Conocer sus estructuras, su dinámica y prioridades, comprender sus tiempos. El desarrollo de este proyecto nos demandó un periodo de adaptación a las otras instituciones participantes que en este caso están más atentas a necesidades concretas del día a día en la problemática sanitaria y educativa de la gente del barrio que a generar a futuro un cambio beneficioso para su gente. La realidad los desborda y el proyecto tuvo que ajustarse a la situación local sin dejar que se desdibuje su objetivo central.

El docente responsable del proyecto "Mujeres enfocando: talleres itinerantes de cine en Villa Soldati", realizado por un equipo de la Facultad de Ciencias Sociales de la Universidad de Buenos Aires, que recibió apoyo del PVU en 2010, quien al responder al cuestionario del proyecto señaló que prefería que solo se citara el nombre del proyecto, señaló lo siguiente: 
En términos generales, la implementación del proyecto permitió problematizar los saberes hegemónicos que circulan acerca de las problemáticas abordadas: salud, violencia de género, prostitución, explotación sexual y trata. A su vez, el desarrollo de los talleres de cine y las producciones audiovisuales realizadas por las organizaciones sociales indican la potencialidad de la herramienta audiovisual para promover procesos de reflexión y diálogo, así como de posicionamiento en la discusión pública. Se abren aquí preguntas acerca de la efectiva implementación de la Ley de Servicios de Comunicación Audiovisual y la participación que finalmente las organizaciones podrán tener en la definición del nuevo mapa comunicacional que esta Ley debería abrir. Particularmente, el proceso abierto durante el taller de cine y la realización del cortometraje "Al gran pueblo, salud!" en el Cesac puso en escena diversas concepciones sobre la salud que habitan la comunidad de referencia; a partir de ello, y de inquietudes previas de integrantes del equipo del Centro, se decidió instalar un "Consultorio Intercultural", integrado por una médica generalista y un curador tradicional del Barrio Fátima, en el cual atienden conjuntamente los procesos de salud-enfermedad que demandan su atención.

Sería posible incluir muchos otros ejemplos ilustrativos, pero con los mencionados parece suficiente para sugerir la diversidad de tipos de aprendizajes que las experiencias objeto de este estudio han facilitado. En contraste con estos logros que contribuyen a mejorar la calidad académica de las universidades, nuestra investigación permitió identificar algunas tensiones institucionales y problemas de comunicación intercultural al interior de las universidades que limitan su mayor desarrollo y valoración por parte de las universidades, las cuales interesa comentar.

\section{TENSIONES INSTITUCIONALES Y PROBLEMAS DE COMUNICACIÓN INTERCULTURAL AL INTERIOR DE LAS UNIVERSIDADES}

La existencia de diferencias significativas al interior de las universidades es una característica no sólo de este tipo de instituciones, sino de todas, o al menos de la mayoría de las organizaciones complejas. En algunas publicaciones anteriores, he ilustrado con ejemplos concretos que las organizaciones complejas no son homogéneas, sino heterogéneas, en el sentido de que a su interior existen y/o se desarrollan diferentes interpretaciones del sentido, visión, misión y valores de las mismas. Así, cabe decir que al interior de organizaciones complejas conviven y contienden diversas culturas institucionales (MATO; MALDONADO; REY,2011; MATO 2012).

Las universidades no son una excepción a esta suerte de regla. Por el contrario, en ellas, cuanto menos debido a la diversidad disciplinar de sus 
miembros y unidades institucionales, tal heterogeneidad puede ser especialmente significativa. A esto se agrega el carácter reflexivo y deliberante que les caracteriza. Así las cosas, no debe resultar sorprendente que al interior de las universidades existan diversas interpretaciones acerca de la función social de la universidad y/o de "la ciencia", relaciones ciencia-otros saberes, relaciones conocimiento teórico y aplicado, relaciones docencia-investigación-extensión u otras modalidades de vinculación con la comunidad.

Significativamente, un buen número de respuestas al cuestionario de nuestro proyecto revelan no sólo la existencia al interior de las universidades de interpretaciones diversas sobre esos asuntos, sino también la preeminencia en ellas de visiones academicistas que no valoran de manera apropiada los tipos de actividades desarrolladas por los proyectos apoyados por el PVU. Este es un asunto directamente relacionado con las diversas "formas de sentido común" respecto del quehacer universitario y la predominancia, y en algunos casos claramente hegemonía, de una de ellas.

Debe destacarse también que las respuestas a los cuestionarios revelan que un buen número de docentes responsables de estos proyectos poseían ya una reflexión elaborada al respecto con anterioridad al desarrollo de las experiencias apoyadas por el PVU. En sus respuestas al cuestionario, algunos de ellos refieren de manera explícita a los estatutos universitarios y/o a ponencias y publicaciones suyas sobre estos asuntos, y explícitamente sientan posición al respecto.

Para ilustrar lo expuesto, resulta interesante citar las respuestas de algunos colegas a una pregunta del cuestionario de nuestro proyecto que inquiría acerca de si en el desarrollo de la experiencia de trabajo con la comunidad a su cargo se habían presentado problemas de comunicación intercultural.

El profesor Guillermo de Hoyos, de la Facultad de Ciencias de la Salud, de la Universidad Nacional del Comahue, a cargo de un proyecto de promoción de la salud que recibió apoyo del PVU en 2008, respondió la pregunta de manera elocuente:"Los únicos que puedo identificar ahora [se relacionan con] una cultura institucional en la que la burocracia administrativa ocupa un lugar central".

En respuesta a la misma pregunta, la profesoraGraciela García Crimi, de la Facultad de Odontología, de la Universidad de Cuyo, responsable del proyecto de "Promoción de la salud buco-dental en sectores socialmente vulnerables del Valle de Uco" sostuvo:

Las dificultades por el contraste de 'visiones de mundo' son garrafales. Los que pretendemos realizar salidas a "campo" no existimos, lo que nosotros hacemos no es importante. Nunca me voy a olvidar de la frase emitida por una de las autoridades de mi Facultad "Pero eso es voluntario, lo hacen porque 
quieren"; respuesta emitida al solicitar permiso para que no se le computara falta a los alumnos que salían ese día a campo por el proyecto.

Otra respuesta significativa es la que ofreció la profesora María Luisa Cacace, de la Cátedra de Microbiología de la Facultad de Ciencias de la Salud de la Universidad Nacional de Salta, responsable de un proyecto del área de salud que recibió apoyo del PVU en 2008, quién respondió en estos términos:

Es necesario que la Universidad transmita sus conocimientos teórico-prácticos y llegue hacia la comunidad en tareas de extensión. No siempre se cumple esta función social, y la mayoría de los investigadores están inmersos en sus tareas de investigación básica, publicaciones, etc., que si bien hace a su curricula, no se aplican estos conocimientos en la resolución directa de problemas sanitarios básicos para la comunidad.

La profesora Rosa María Sims, de la Universidad Nacional de Tucumán, responsable del proyecto "Germinando un modelo de red social para la protección de niños que nacieron en condiciones de riesgo", que recibió apoyo del PVU en 2010, apuntó.

Estamos todavía exigidos de algún modo al éxito académico, a los trabajos cientificos en congresos y a los premios, como evaluación curricular. Este tipo de proyectos, por ejemplo, no aporta al docente ni al estudiante en términos de créditos una valoración equivalente, muy por el contrario es mucho el tiempo y esfuerzo invertido que no tiene correspondencia en la apreciación final en un concurso en las ciencias de la salud, por ejemplo. El problema más serio que veo en esto, es la tácita ubicación de valores humanos en un plano inferior. Ser solidario lleva tiempo, irónicamente es tiempo lo que necesito para ser exitoso y hacer una carrera académica. Entonces, queda liberado al docente y del alumno la elección

El profesor César EnriqueSirka, de la Facultad de Recursos Naturales (FRN) de la Universidad Nacional de Formosa, responsable de un proyecto de producción agropecuaria y forestal, que recibió apoyo del PVU en 2010, señaló

Lo que queda pendiente es darle a este tipo de actividades como las propuestas dentro del programa de voluntariado similar ponderación que los trabajos de investigación para los docentes, aspecto este que preocupa al grupo de docente participantes y que ha originado la presentación por el suscripto al Concejo Directivo de la F.R.N para que considere de interés académico las acciones desarrolladas dentro del programa, que significaron actividades de interacción entre docentes de diferentes cátedras, capacitación a alumnos universitarios, docentes $y$ alumnos de escuelas rurales con fuerte contenido práctico, formalización de convenios entre organismos y la efectiva inserción de la universidad para atender ciertas problemática en su medio. 
El profesor Fabián Grosman, de la Universidad Nacional del Centro de la Provincia de Buenos Aires, responsable del proyecto "Conocer para valorar y respetar. El arroyo del Azul desde la perspectiva ambiental", que recibió apoyo del PVU en 2010, expuso lo siguiente:

Considero de la misma relevancia y jerarquía la extensión universitaria, tanto como la docencia y la investigación; creo que en esta etapa de necesidades nacionales estamos en una política de investigación que no apunta a reforzar los requerimientos sociales o dar respuesta real y concreta a problemáticas complejas para lo cual está capacitada, sino que hemos pauperizado el sistema y medido todo a partir del número de trabajos en revistas extranjeras.

La ya mencionada Dra. Betina Cecilia Pezzani, de la Cátedra de Microbiología y Parasitología de la Universidad Nacional de La Plata, a cargo de un proyecto que recibió apoyo del PVU en 2010, sostuvo:

Dentro de la propia estructura de la cátedra hemos encontrado resistencia a nuestra participación en dichos emprendimientos. Muchas veces hemos sido subestimadas como docentes-investigadoras por docentes superiores y pares de nuestro lugar de trabajo por presentarnos en las distintas convocatorias, percibimos ese dejo de no estar haciendo algo valioso como escribir un paper o ir a un Congreso, que se perdía el tiempo. De hecho se nos ha preguntado personalmente si queríamos dejar de hacer investigación. Muy por el contrario nuestra producción científica no mermó y hemos podido organizar todas las tareas sin desmedro de nuestras funciones como docentes con dedicación exclusiva. Nada nos frenó, muy por el contrario nos reforzó la idea que la Universidad tiene que estar con todos sus recursos humanos, docentes y estudiantes, cerca de la gente, escuchando, conteniendo, asistiendo y aportando soluciones a problemas concretos que en la mayoría de los casos surgen de la propia comunidad. Nuestro proyecto articuló armónicamente la extensión con la docencia y la investigación. Seguiremos participando en todo proyecto que contemple nuestro compromiso con la comunidad.

Limitaciones de extensión impiden citar más respuestas a los cuestionarios, pero generalizando puede afirmarse que permiten observar la existencia al interior de las universidades de diferentes interpretaciones respecto de su papel en la sociedad y cómo cumplirlo. También dan cuenta del hecho que las visiones predominantes no son precisamente las que valoran el tipo de proyectos de trabajo con la comunidad y que existen obstáculos para desarrollar proyectos con grupos de población y sus organizaciones. Adicionalmente, muchas de ellas ilustran cómo en general, las actividades de vinculación con comunidades no son tan fomentadas como las de investigación, y en muchos casos ni siquiera 
son consideradas en la evaluación de los docentes. La investigación realizada lleva a concluir que esto aún es así, incluso pese a que en los últimos años en Argentina han ocurrido interesantes avances en la valoración institucional de las labores de extensión universitaria y vinculación social, tal como lo apuntan en sus respuestas a los cuestionarios varios de los responsables de los proyectos que recibieron apoyo del PVU.

Por ejemplo, desde 2003 existe un programa específico de la Secretaría de Políticas Universitarias (SPU) del Ministerio de Educación orientado a proveer fondos para Proyectos de Vinculación y Extensión en Universidades Nacionales(Resolución SPU No 97/2003). Adicionalmente, en 2012 el Ministerio de Educación emitió la Resolución 692, mediante cuyo artículo 1 se insta a las universidades e Instituciones de Educación Superior públicas y privadas a que a efectos del desarrollo de la carrera docente universitaria, otorguen una valoración específica y positiva a quienes "además del dictado de clases desarrollen tareas de investigación, extensión, vinculación y transferencia de conocimiento, guía o acompañamiento en las acciones de voluntariado que realicen los estudiantes". A esas resoluciones ministeriales se agrega que el Consejo Interuniversitario Nacional (CIN), órgano público no estatal creado en 1985 como organismo coordinador de las políticas universitarias, que nuclea a las universidades que voluntariamente adhirieran a él, en septiembre de 2009, emitió el documento titulado "Lineamientos para un Programa de Fortalecimiento de la Extensión Universitaria en las Universidades Públicas Argentinas (Acuerdo Plenario Nro. 711/09)" mediante el cual busca fomentar la misma. Dicho documento da cuenta, entre otras cosas, de que desde la creación misma del organismo éste ha contado con una Comisión de Extensión y que desde 1992 ha producido varios acuerdos plenarios orientados a valorizar las actividades de extensión. El mencionado documento de Lineamientos concluye formulando una Propuesta de Desarrollo de un Programa de Fortalecimiento de la Extensión Universitaria en las Universidades Públicas, la cual entre otros criterios compartidos por las universidades nacionales menciona el de "Constitución de saberes en forma conjunta con las comunidades". Al respecto, cabe apuntar que esta manera de entender la "Extensión Universitaria" caracteriza a varios de los proyectos que hemos analizado en la investigación que da lugar al presente artículo, y que además se corresponde con la idea de "colaboración intercultural" en la producción de conocimientos expuesta en estas páginas y en publicaciones anteriores asociadas al proyecto sobre diversidad cultural e interculturalidad en educación superior (MATO, 2008a, MATO, 2009c). 


\section{OBSTÁCULOS A LA VINCULACIÓN SOCIAL Y COLABORACIÓN INTERCULTURAL EN EDUCACIÓN SUPERIOR}

Los recientes avances institucionales y de políticas respecto de la extensión universitaria y la vinculación social de las universidades antes señalados, acompañan visiones y posturas que de un modo u otro pueden observarse en casi todas las universidades públicas argentinas, algunas con siglos de antigüedad, otras con apenas unas décadas o incluso sólo unos años de existencia. Todas ellas, cada una a su manera, parecen estar avanzando hacia otorgar mayor valoración a las labores de extensión, así como en repensar la idea de extensión universitaria. Sin embargo, las respuestas a los cuestionarios de esta investigación llevan a concluir que en la mayoría de las universidades estos movimientos relativamente recientes, aún no resultan suficientes para cambiar significativamente un modelo de universidad que lleva siglos hegemónicamente instalado como parte de la cultura institucional universitaria, no sólo en Argentina, sino en el mundo.

Se trata de un modelo de universidad academicista cuya escasa valoración por la vinculación social, en las últimas décadas, se ha visto fortalecido por los factores mencionados al comienzo de este artículo. Es decir, por ciertas políticas de estímulo a la investigación que evalúan logros preeminentemente mediante publicaciones, citas y patentes, así como por la creciente ascendencia de los rankings internacionales de universidades en la opinión pública en general y particularmente en algunos sectores universitarios, así como en otros ámbitos muy significativos, como los de formulación de políticas universitarias y los de tomadores de decisiones presupuestarias.

En contraste con la preeminencia de ese modelo y la importancia de los mencionados factores, la investigación realizada permite concluir que las experiencias de colaboración entre equipos universitarios y comunidades y organizaciones sociales, no sólo contribuyen a mejorar la calidad de vida de sectores sociales, sino también la formación profesional ofrecida por las universidades, así como las posibilidades y calidad de investigación que realizan dichos equipos. Sin embargo, estos tipos de actividades no suelen contar con valoración institucional. Esto hace aconsejable que las universidades y los organismos de evaluación integren estas modalidades de trabajo como un componente importante de los planes de estudio, y le otorguen mayor valoración en sus políticas de investigación y de carrera docente.

Si bien, esta investigación toma como referencia algunas experiencias en universidades argentinas, ni las ventajas de las experiencias de vinculación social universitaria referidas, ni los problemas de valoración institucional por 
las mismas, se limitan a ese país. Respecto de que las ventajas de esos tipos de experiencias no se limitan a ese país, en páginas precedentes se han expuesto las conclusiones de una investigación enfocada en el estudio de aproximadamente cuarenta experiencias de educación superior orientadas principalmente a responder a demandas y propuestas de comunidades y organizaciones indígenas y afrodescendientes de once países de América Latina anteriores, algunas de las cuales corresponden a programas especiales desarrollados en universidades "convencionales" y otras a universidades u otras instituciones concebidas como "interculturales" (MATO, 2011; MATO, 2008a, 2009b, 2009c). Por otra parte, el problema de subvaloración por las experiencias de extensión y vinculación social universitaria, tampoco es exclusivo de ese país, pues se enmarca en algunas tendencias y procesos de alcance internacional, que se expresan en ciertas culturas institucionales, en algunas políticas de estímulo a la investigación, evaluada en términos de publicaciones, citas y patentes, y en la creciente ascendencia de los rankings universitarios en la opinión de actores sociales muy significativos para el tema que nos ocupa(DIAS SOBRINHO; BRITO DIAS, 2006;LÓPEZ SEGRERA, 2008;ORDORIKA, 2007).

Para valorar más apropiadamente su importancia, es necesario destacar que estos problemas relativamente nuevos se asientan en otros de más larga data y que hacen a la hegemonía de ciertas ideas de universidad y de ciencia, sobre cuya profundidad, alcance, y consecuencias, es necesario argumentar.

No se trata simplemente de una coyuntural orientación de políticas, sino de algo menos fácil de cambiar, "una manera de ver las cosas", un cierto "sentido común" y con ellos una cierta episteme. En los contextos institucionales en los cuales se producen y reproducen representaciones y políticas de educación superior, ciencia y tecnología (incluyendo agencias gubernamentales dedicadas a estos temas, universidades e institutos nacionales de investigación), las referencias explícitas o implícitas a la existencia de dos clases de saber, uno solo de los cuales tendría validez universal, mientras que el otro (diverso a su interior) no la tendría, resultan hegemónicas. Esas representaciones, que expresan una cierta visión de mundo, se han convertido en "sentido común", y es partir de ellas que se formulan la mayoría de las políticas y normas que regulan los campos de educación superior y ciencia y tecnología. Más aún, frecuentemente este sistema de representaciones incluye una cierta visión de la ciencia según la cual existen unas "ciencias duras" y otras que por contraposición no quedaría más alternativa que llamar "blandas".

Pensar la producción y validez de conocimiento divididas en dos mundos, uno solo de los cuales sería poseedor de verdades "universales" y el otro de 
verdades que, en contraposición con la postulada y pretendida universalidad de aquéllas, sólo cabría asumir como "locales", es tan antigua como el credo en la superioridad del "hombre blanco" y la "civilización occidental", que pretendidamente sería la generadora y poseedora de tal saber pretendidamente "universal". Los encuentros, conflictos y negociaciones entre ese saber supuestamente "universal" y esos otros en "locales", se han hecho más frecuentes en las últimas décadas por la creciente extensión y profundización de relaciones entre actores sociales que desarrollan sus prácticas a escala mundial, o en algunos casos continental o regional, y los que las desarrollan a escalas más "locales", sean estas nacionales, provinciales, municipales o comunitarias. El análisis de las limitaciones y consecuencias de la creencia en la existencia de un saber pretensiosa y pretendidamente "universal" y otros de validez apenas "local" resulta no sólo más necesario, sino también más factible, en el marco de los procesos de globalización contemporáneos, debido a la creciente importancia de los intercambios entre actores sociales cuyas maneras de ver del mundo, producir conocimiento y accionar, se forman en muy diversos contextos y dan lugar a muy diversos tipos de saber. Por ello, la colaboración intercultural tanto en la producción de conocimientos, como en la formación de profesionales y técnicos se hace cada día más necesaria y también más factible (MATO, 2008a).

Sin embargo, a pesar de los ya no tan recientes análisis y argumentaciones acerca los condicionantes históricos y contextuales del conocimiento científico (por ejemplo, algunos que ya han devenido "clásicos": BOURDIEU, 1988, 2001; FOUCAULT, 1966, 1979; KUHN, 1971, 1987), podemos observar que las prácticas y discursos de numerosos investigadores, instituciones de investigación y formación académica y organismos de formulación de políticas de educación superior, ciencia y tecnología, se asientan, cuanto menos implícitamente, en la idea de que "la ciencia", como modo de producción de conocimientos, y el "conocimiento científico", como acumulación de conocimientos producidos "científicamente" tendrían validez "universal". Es decir, resultarían verdaderos y aplicables en cualquier tiempo y lugar. En el marco de esa visión de mundo, la otra clase abarcaría una amplia diversidad de tipos de saber, de modos de producción de conocimiento y sus resultados, a los cuales, en contraposición con el saber "universal" de la "ciencia", suele caracterizarse, según los casos, como "étnicos" o "locales", en cualquier caso como saberes "particulares", es decir "no-universales". Dentro de tal visión de mundo, estos "otros" saberes sólo tendrían validez y aplicación "local", al menos hasta tanto sean validados con los métodos propios de "la ciencia". 
Un ejemplo significativo de los problemas que esto plantea es la evaluación y validación de conocimientos "étnicos" y otros calificados simplemente como "locales" sobre las aplicaciones terapéuticas de especies vegetales, mediante métodos "científicos", a lo que, significativamente, suele seguir su apropiación y patentado por instituciones "científicas" y/o más frecuentemente laboratorios farmacéuticos (BLUM, 1993; BRUSH, 1993; GREEN, 2004; NIGH, 2002). Semejante efecto práctico de esta división no puede pasarse por alto, como tampoco puede pasarse por alto que en las universidades la investigación y docencia sobre, por ejemplo, medicina e instituciones políticas, jurídicas y económicas, propias de esos "otros" grupos humanos suelan quedar reservadas a los departamentos y escuelas de Antropología y sólo de manera excepcional sean materia de investigación y formación en departamentos y escuelas de Derecho, Ciencias Políticas, Economía, Medicina, o de las que vengan al caso según el tipo de saber en cuestión.

En los últimos años han surgido voces que postulan que con el propósito de construir sociedades más "incluyentes" las universidades deberían ofrecer formación en esos "otros" saberes, pero frecuentemente esto se ve en términos de concesión a esos "otros" grupos humanos. Esta visión condescendiente pierde de vista que la colaboración intercultural resulta imprescindible tanto para quienes formamos parte de las instituciones que producen conocimiento cuyo valor supuestamente tendría carácter "universal" (las universidades y otros centros de producción de conocimiento "científico"), como para quienes desarrollan sus prácticas en otros tipos de marcos institucionales y sociales y producen conocimientos que suelen calificarse como "locales" o "particulares". Pensar la idea de colaboración intercultural en educación superior y en ciencia y tecnología, y en conexión con esto en la incorporación de otros saberes y formas de producción de conocimientos, como una condescendiente política de "inclusión" resulta pobre y auto-empobrecedoramente racista. No se trata de hacer concesiones, sino de reconocer las limitaciones de esas visiones racistas para poder superar el monoculturalismo, y desarrollar perspectivas de investigación y de formación de profesionales y técnicos que sean pertinentes con la diversidad cultural que caracteriza a las sociedades contemporáneas (MATO, 2008a). En una serie de libros colectivos producto de la investigación precedente ya mencionada se han documentado las características de unas cuarenta experiencias desarrolladas en once países latinoamericanos que constituyen avances en esa dirección (MATO, 2008a, 2009b, 2009c).

El problema que se confronta no es simplemente de políticas universitarias, es más profundo. Se relaciona con la hegemonía de valores que operan 
como creencias entre no pocos científicos "serios", docentes universitarios y autoridades y tomadores de decisiones en este campo. Un ejemplo saliente lo constituyen las pretensiones de objetividad del conocimiento científico y la neutralidad de valores de los investigadores. La aplicación de estas ideas, provenientes de las llamadas "ciencias duras", a las llamadas "ciencias sociales", conduce a ignorar cómo nuestra subjetividad es constitutiva de nuestro trabajo de investigación. La pretensión de objetividad supone ante todo cerrar los ojos a algunas formas ineludibles de subjetividad, como por ejemplo la que necesariamente afecta al planteamiento de un problema, la formulación de las preguntas que han de guiar la investigación, el desarrollo de una perspectiva de análisis y el establecimiento de relaciones con las personas cuyas prácticas estudiamos. Todo esto de ningún modo puede ser "objetivo", al menos no en las llamadas ciencias sociales. Todo esto depende de desde dónde se investiga, para qué y qué se pretender hacer con los resultados de la investigación. Pero estas preguntas no siempre se plantean. Antes bien, la investigación funciona de manera compulsiva respecto de ellas, es decir de manera no reflexionada. Las respuestas están allí instaladas con la firmeza y "naturalidad" de un credo, de modo tal que este tipo de preguntas no se formulan, sino excepcionalmente.

El problema que quienes impulsan las concepciones cientificistas parecen no comprender es que tanto las propias preguntas de investigación, como los modos de producción de datos, dependen en última instancia de opciones epistemológicas, las cuales están asociadas a visiones de mundo y posiciones éticas y políticas que dependen entre otros factores del tipo de relaciones que se sostiene o se aspira a sostener con actores sociales extra-académicos. Las posiciones éticas y políticas son constitutivas del basamento epistemológico y de las perspectivas teóricas de nuestras investigaciones; y así también de las preguntas y de los métodos.

Las preguntas de investigación no son las mismas, ni tampoco los métodos, si lo que se pretende es "escribir" estudios supuestamente "objetivos", que si se pretende producir algún tipo de saber útil a los intereses de actores sociales extra-académicos. De las respuestas a preguntas del tipo ¿para qué y para quién/es investigar? depende qué investigar, cómo, con quiénes, en el marco de cuáles relaciones, con cuáles propósitos. De tales respuestas también dependen decisiones tales como si la investigación en cuestión acabará en una publicación académica o en qué "cosa" (un video, un museo, un programa de acción comunicativa, educativa, una experiencia de organización social, un plan de salud, un emprendimiento productivo, etc.) y cómo pensamos que tales 
"cosas" deberían o podrían circular y/o ser útiles, a quiénes, qué importancia tendrían los resultados y cuál los procesos/experiencias.

El caso es, entonces, que el cientificismo acarrea dos tipos de problemas a las universidades. Por un lado, obstaculiza que otros modos de conocimiento y las prácticas profesionales extra-académicas puedan ser objeto de mejor valoración y cuidadosa articulación y aprovechamiento en la investigación y formación universitarias. Por otro lado, acaba por afectar la pertinencia y legitimidad social de la formación y prácticas universitarias, así como por excluir oportunidades de intercambio, aprendizaje y participación en algunas dinámicas sociales. En contraste con esto, las modalidades de colaboración entre equipos universitarios y diversos sectores de población pueden contribuir a mejorar la calidad de vida de la población, así como la formación de profesionales y el desarrollo de investigación y aplicaciones. Las más de doscientas experiencias que sirven de referencia a este artículo constituyen ejemplos de ello.

Así, cabe afirmar que la investigación en que se basa este artículo lleva a concluir que no solo por razones sociales, sino también por razones académicas, tanto para mejorar la calidad de la formación profesional que ofrecen, como para mejorar la calidad de la investigación que realizan, parece necesario que las universidades ajusten su valoración por las posibilidades que ofrece el trabajo directo de equipos universitarios con grupos de población y sus organizaciones. $\mathrm{Y}$, en consecuencia, que integren esta modalidad de trabajo como un componente importante de los planes de estudio, y le otorguen mayor valoración en sus políticas de investigación y de carrera docente. Pero la puesta en práctica de esta orientación tropieza con tensiones institucionales y problemas de comunicación intercultural dentro de las universidades como los señalados anteriormente.

\section{REFERENCIAS}

BLUM, Elissa. Making biodiversity conservation profitable.A case study of the Merck/INBio Agreement.Environment,Washington,v.35, n. 4, p. 16-45, 1993.

BOURDIEU, Pierre .Homo Academicus. Stanford: StanfordUniversity Press. 1988.

BOURDIEU, Pierre. El oficio del científico. Barcelona: Anagrama. 2001. 
BRUSH,Stephen B. Indigenous knowledge of biological resources and intellectual property rights: the role of anthropology. American Anthropologist, Washington,v. 95, n. 3, p. 653-671, 1993.

CUCHE, Denys.La noción de cultura en las ciencias sociales. Buenos Aires: Ediciones Nueva Visión,1999.

DIAS SOBRINHO, José; DIAS, Rafael de Brito. Acreditação da Educação Superior e C\&T: políticas e ideologia. Avaliação, Campinas, v. 11, n.4, p. 9-25, mar. 2006.

FOUCAULT, Michel. EI nacimiento de la clínica: una arqueología de la mirada médica.México: Siglo XXI, 1966.

FOUCAULT, Michel. Las palabras y las cosas: una arqueología de las ciencias humanas.México: Siglo XXI, 1979.

GARCÍA CANCLINI, Néstor. Diferentes, desiguales y desconectados. Barcelona: Gedisa, 2004.

GREENE, Shane.Indigenous people incorporated? Culture as politics, culture as property in pharmaceutical bioprospecting.CurrentAnthropology, Chicago, v. 4, n. 2, p. 211-224,2004.

ENCUENTRO de Redes Universitarias y Consejos de Rectores de América Latina y el Caribe, 4., 2011, Buenos Aires. Posición de américa latina y el Caribe ante los rankings de la educación superior. Buenos Aires, 2011.

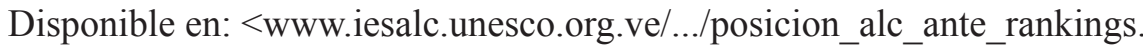
pdf>. Acceso en: 9 oct. 2012.

KUHN, Thomas La estructura de las revoluciones científicas. México: FCE, 1971.

KUHN, Thomas. La tensión esencial. México: FCE, 1987.

LÓPEZ SEGRERA, Francisco. Tendencias de la educación superior en el mundo y en américa latina y el Caribe. Avaliação, Campinas; Sorocaba, v.13, n.2,p. 267-291, 2008.

MATO, Daniel. Cultura, comunicación y transformaciones sociales en tiempos de globalización. En: MATO, Daniel; MALDONADO, Alejandro (Comps.).Cultura y transformaciones sociales. Perspectivas latinoamericanas. Buenos Aires: CLACSO, p. 13-84, 2007. 
MATO, Daniel. No hay saber "universal": la colaboración intercultural en la producción de conocimientos es imprescindible. Alteridades,Iztapalapa, v.18, n. 35, p.101-116, 2008a.

MATO, Daniel(Coord.). Diversidad cultural e interculturalidad en educación superior. Experiencias en américa latina. Caracas: IESALCUNESCO, 2008b.

MATO, Daniel. Contextos, conceptualizaciones y usos de la idea de interculturalidad. En: AGUILAR, Miguel Ángel; NIVÓN, Eduardo; PORTAL, María Ana; WINOCUR, Rosalía (Coords.). Pensar lo contemporáneo: de la cultura situada a la convergencia tecnológica. Barcelona; México: Anthropos Editorial; Universidad Autónoma Metropolitana, 2009a.

MATO, Daniel (Coord.).Instituciones interculturales de educación superior en américa latina. Procesos de construcción, logros, innovaciones y desafíos. Caracas: IESALC-UNESCO, 2009b.

MATO, Daniel (Coord.). Educación superior, colaboración intercultural y desarrollo sostenible/buen vivir. Experiencias en América Latina. Caracas: IESALC-UNESCO, 2009c.

MATO, Daniel. Forms of intercultural collaboration between institutions of higher education and indigenous and Afro-descendant peoples in Latin America. Postcolonial Studies, Routledge, v. 14, n. 3, p. 331-346, 2011.

MATO, Daniel. Socio-cultural differences and intercultural communication in social participation experiences Journal of Intercultural

Communication Studies, United States, v.21, n.1, p. 101-116,2012.

MATO, Daniel; MALDONADO Alejandro; REY, Enrique.

Interculturalidad y comunicación intercultural. Propuesta teórica y estudio de experiencias de participación social en la gestión de servicios públicos en una comunidad popular de la ciudad de Caracas.Caracas: Universidad Central de Venezuela, 2011.

NIGH, Ronald.Maya Medicine in the Biological Gaze Bioprospecting Research as Herbal Fetishism.CurrentAnthropology, Chicago, v. 43, n. 3, p. 451-464,2002.

ORDORIKA, Imanol. Universidades y globalización: tendencias hegemónicas y construcción de alternativas. Revista Educación Superior y Sociedad, Caracas, v. 12, n. 1, p. 175-190, 2007. 